Fileti, Coutinho and Canuto, Adv. Quantum Chem., 47, 51 (2004).

\title{
Is There a Favorite Isomer for Hydrogen-Bonded Methanol in Water?
}

\author{
Eudes E. Fileti ${ }^{1}$, Kaline Coutinho ${ }^{2}$ and Sylvio Canuto ${ }^{1}$ \\ ${ }^{1}$ Instituto de Física, Universidade de São Paulo, \\ CP 66318, 05315-970, São Paulo, SP Brazil. \\ ${ }^{2}$ Universidade de Mogi das Cruzes/CIIB, \\ CP 411, 08701-970, Mogi das Cruzes, SP, Brazil.
}

October 15, 2003

\begin{abstract}
Sequential Monte Carlo/Quantum Mechanical calculations of the interaction energy of hydrogen-bonded methanol in liquid water gives the same result for methanol acting either as the proton donor or the proton acceptor. For the complex-optimized cases methanol acting as the proton acceptor, $\mathrm{CH}_{3} \mathrm{HO} \cdots \mathrm{H}_{2} \mathrm{O}$, is more stable than the proton donor, $\mathrm{CH}_{3} \mathrm{OH} \cdots \mathrm{OH}_{2}$, by $\sim 0.5 \mathrm{kcal} / \mathrm{mol}$. In the case of methanol in liquid water, at room temperature, statistically converged results, using counterpoise corrected MP2/aug-cc-pVDZ calculations, leads to the same binding energy in both cases.
\end{abstract}




\section{Contents}

1 Introduction 3

2 Methods $\quad 4$

2.1 Monte Carlo Simulation . . . . . . . . . . . . . . 4

2.2 Hydrogen Bonds from Monte Carlo Simulation . . . . . . . . . 5

2.3 Quantum Mechanical Calculations . . . . . . . . . 8

$\begin{array}{llr}3 & \text { Results } & \mathbf{9}\end{array}$

3.1 Gas Phase Interaction . . . . . . . . . . . . . . . . . . . 9 9

3.2 Liquid (1:1) Interaction . . . . . . . . . . . . . . . . . 10

3.3 Statistical Convergence . . . . . . . . . . . . . 12

4 Final Remarks and Conclusions $\quad 12$ 


\section{Introduction}

Alcohol-water liquids are among the most interesting liquid mixtures[1, 2, 3]. Indeed, alcohol-water exhibits properties that are of great interest in physics, chemistry and biology. To a large extent this is a consequence of the great ability of both water and alcohol to make hydrogen bonds. They are both proton donor and proton acceptor of hydrogen bonds. The methanol-water system can have two hydrogen-bonded structures corresponding to the two possible heterodimers (or isomers) where methanol is a proton acceptor, $\mathrm{CH}_{3} \mathrm{HO} \cdots \mathrm{H}_{2} \mathrm{O}$, or a proton donor, $\mathrm{CH}_{3} \mathrm{OH} \cdots \mathrm{OH}_{2}$, of the hydrogen bond (see figure 1). The existence of these two isomers is easy to realize and the strengths of the two possible interactions are of great interest. In fact they are related to several thermodynamic and physico-chemical properties. For instance, the mixture of methanol and water exhibit an entropy value that is considerably smaller than one should expect $[1,4,5]$. Also there are enormous consequences in the segregation and in the hydrophobic role of the $\mathrm{CH}_{3}$ group leading to fundamental questions regarding the orientational local order $[4,5]$. Methanol-water is one of the simplest examples of the amphoteric relation because both methanol and water can act as proton donor and proton acceptor of hydrogen bonds. The question of which of the two isomers is the more stable is conspicuous. Early theoretical studies have obtained controversial results $[6,7,8,9,10]$ regarding the relative stability of the two isomers. Even experimental results have missed the $\mathrm{CH}_{3} \mathrm{OH} \cdots \mathrm{OH}_{2}$ isomer and obtained only one heterodimer[11]. It was not until very recently that the question has been confidently answered for the gas phase complexes. Detailed microwave rotation-tunneling spectroscopy[12] has set the issue and established that the complex where methanol is the proton acceptor and water is the proton donor, $\mathrm{CH}_{3} \mathrm{HO} \cdots \mathrm{H}_{2} \mathrm{O}$ in figure 1 (left), is the more stable isomer in gas phase. More recent theoretical calculations have indeed confirmed the greater stability of this isomer but obtained a relative stability that is less than $1 \mathrm{kcal} / \mathrm{mol}[13,14]$. Although gas phase results are of interest in their own, it is also clear that the liquid situation may find a broader range of interest for alcohol-water mixtures[15, 16, 17, 18]. Unfortunately, the results obtained for gas phase cannot be directly extrapolated to the liquid situation without a great risk. The hydrogen bonds in a liquid environment certainly do not satisfy a minimum-energy structure. In fact, a liquid is better described by statistical physics and its structure is represented by a great number of possible configurations or molecular arrangements. The thermal 
contribution leads to a decrease of the hydrogen bond interaction[19, 20]. Hence, the strength of the hydrogen bond formed between methanol and water in gas phase is not the same as in the liquid case. The focus of this present contribution is to clarify this aspect. In fact, we will make an attempt to obtain the relative strength of the two hydrogen-bonded isomers formed between methanol and water in the liquid environment at room temperature. We use Monte Carlo simulation to generate the structure of the liquid composed of methanol in bulk water. Using the structures of the simulation, we identify the hydrogen bonds in the liquid, in both isomeric possibilities, select the configurations and perform ab initio quantum mechanics calculations to obtain the intermolecular strength. These results clarify the relative strengths in the liquid environment and also give a theoretical estimate of the decrease of the hydrogen bond interaction as compared to the gas phase situation.

\section{Methods}

\subsection{Monte Carlo Simulation}

The structure of the liquid is generated by Metropolis Monte Carlo (MC) computer simulation[21], using the DICE Monte Carlo program[22]. The simulations are performed in the canonical $(N V T)$ ensemble with one methanol molecule plus 400 water molecules using the experimental density of water, which at $T=298.15 \mathrm{~K}$ is $0.9966 \mathrm{~g} / \mathrm{cm}^{3}$. The intermolecular interactions are described by the standard Lennard-Jones plus Coulomb potentials. For the water molecules we use the SPC potential[23] and for methanol we use the all-site OPLS-AA potential[24]. Our sequential Monte Carlo/quantum mechanics procedure for generating liquid structure using $\mathrm{MC}$ simulation has been described previously in great detail[19, 20, 25, 26]. The present simulation consisted of an equilibration stage of $2.0 \times 10^{6} \mathrm{MC}$ steps, followed by a long averaging stage of $80.0 \times 10^{6} \mathrm{MC}$ steps, where the equilibrium configurations are generated. Successive configurations generated in the MC simulations, are found to be statistically highly correlated, and will not give important additional information. Therefore we calculate the interval of statistical correlation using the auto-correlation function of the energy[27]. For the simulations presented here, after the calculation of the auto-correlation of the energy we obtain that configurations separated by $4.0 \times 10^{5} \mathrm{MC}$ steps 
are statistically relevant ( $\sim 20 \%$ of statistical correlation). Thus, after the entire simulation, 200 configurations separated by $4.0 \times 10^{5} \mathrm{MC}$ steps were sampled.

\subsection{Hydrogen Bonds from Monte Carlo Simulation}

An important point is the identification of the hydrogen bonds in the structures generated in the Monte Carlo simulation. Hydrogen bonds are normally extracted from computer simulation using the pair-wise radial distribution function[21]. This gives the coordination of water molecules around the solute, but it cannot be assured that all near-neighbor water molecules are indeed hydrogen-bonded. This point has been the subject of previous concern[19, 20, 26, 27, 28]. The definition of a hydrogen bond in a liquid

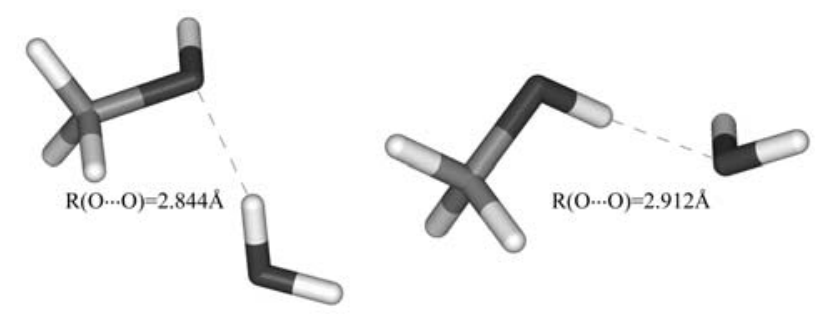

Figure 1: The two isomers of hydrogen-bonded methanol-water.

is not unanimously free from ambiguities. A very efficient procedure however can be obtained using in addition to the geometric, also an energetic criterion[19, 20, 26, 29, 30, 31]. Here we identify a hydrogen-bonded structure when the distance $R_{O-O} \leq 3.35 \AA$, the angle $\theta(O-O H) \leq 40^{\circ}$ and the binding energy is larger than $2.5 \mathrm{kcal} / \mathrm{mol}$. These geometric conditions are obtained from the radial and angular distribution functions. For the energetic criterion we look at the pair-wise energy distribution.

First, figure 2 shows the pair-wise radial distribution function between the $\mathrm{O}$ atom of methanol and the $\mathrm{O}$ atom of water. The first peak in this $G_{O-O}(r)$ distribution function starts at $2.45 \AA$ and ends at $3.35 \AA$, with a maximum at $2.75 \AA$. Hydrogen bonds can therefore be located for the distance $R_{O-O} \leq 3.35 \AA$. Similar analysis gives the criterion for the angle $\theta(O-O H) \leq 40^{\circ}$. Figure 3, in turn, identifies the hydrogen bonds for pairwise methanol-water interaction energy less than $-2.5 \mathrm{kcal} / \mathrm{mol}$. Using these three criteria, in the $200 \mathrm{MC}$ configurations we find 287 hydrogen bonds 


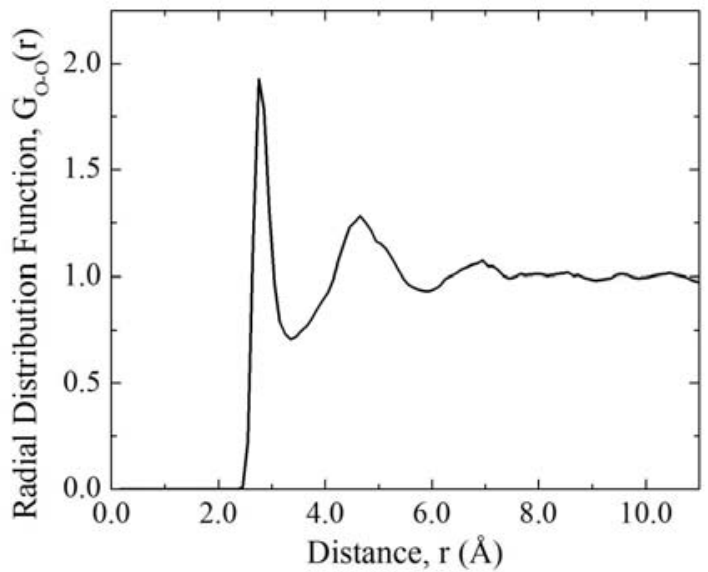

Figure 2: Pairwise radial distribution function between the oxygen atoms of methanol and water.

formed in the $\mathrm{CH}_{3} \mathrm{HO} \cdots \mathrm{H}_{2} \mathrm{O}$ and 160 in the $\mathrm{CH}_{3} \mathrm{OH} \cdots \mathrm{OH}_{2}$ isomers. Table 1 shows the detailed statistics of hydrogen bonds. For the $\mathrm{CH}_{3} \mathrm{HO} \cdots \mathrm{H}_{2} \mathrm{O}$ isomer we find that $7(3.5 \%)$ of the configurations do not form any hydrogen bonds, in 103 (51.5\%) it forms one, and in $86(43 \%)$ it forms two and in only $4(2 \%)$ it forms three hydrogen bonds. This gives an average of 1.43 hydrogen bond between methanol and water in the $\mathrm{CH}_{3} \mathrm{HO} \cdots \mathrm{H}_{2} \mathrm{O}$ isomer. For the $\mathrm{CH}_{3} \mathrm{OH} \cdots \mathrm{OH}_{2}$ the picture is slightly different and $40(20 \%)$ configurations make no hydrogen bond and the rest, 160 (80\%) makes one hydrogen bond, leading to an average of 0.80 hydrogen bond. Because of the

Table 1: Statistics of the hydrogen bonds formed between methanol and water. See text.

\begin{tabular}{ccc}
\hline \hline Number of hydrogen bonds & $\mathrm{CH}_{3} \mathrm{HO} \cdots \mathrm{H}_{2} \mathrm{O}$ & $\mathrm{CH}_{3} \mathrm{OH} \cdots \mathrm{OH}_{2}$ \\
\hline 0 & $7(3.5 \%)$ & $40(20.0 \%)$ \\
1 & $103(51.5 \%)$ & $160(80.0 \%)$ \\
2 & $86(43.0 \%)$ & - \\
3 & $4(2.0 \%)$ & - \\
Average & 1.43 & 0.80 \\
\hline \hline
\end{tabular}




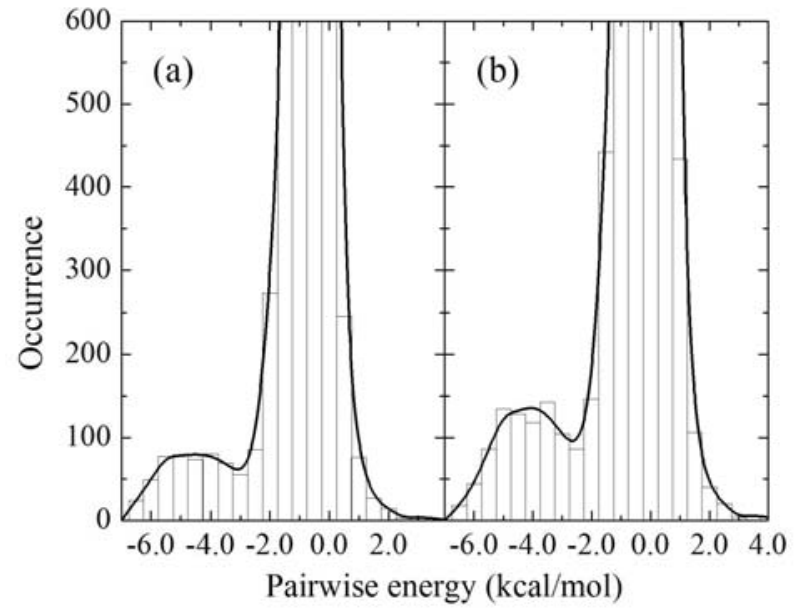

Figure 3: Histogram of the pairwise energy interaction between methanol and water. (a) is the $\mathrm{CH}_{3} \mathrm{OH} \cdots \mathrm{OH}_{2}$ isomer and (b) is the $\mathrm{CH}_{3} \mathrm{HO} \cdots \mathrm{H}_{2} \mathrm{O}$.

great diversity of structures of methanol-water mixture[18] an explanation is needed. The physical situation considered here is methanol in extreme dilution in water. Hence, only one methanol molecule is considered. In a liquid mixture with proper proportion there will also be configurations where two methanol molecules acting as proton donor are hydrogen-bonded to a single water molecule. This, however, will not be present here giving a more simplified picture and a more direct comparison with the (1:1) gas phase result. Thus we will focus in the 103 configurations making one hydrogen bond in the $\mathrm{CH}_{3} \mathrm{HO} \cdots \mathrm{H}_{2} \mathrm{O}$ and 160 configurations in $\mathrm{CH}_{3} \mathrm{OH} \cdots \mathrm{OH}_{2}$. All these $(1: 1)$ structures composed of one methanol and one water molecule will be submitted to the quantum mechanical calculations. In fact statistical convergence is obtained before we use all these hydrogen-bonded structures.

Figure 4 shows, in a single picture, the superposition of all these (1:1) hydrogen bond structures in the liquid, for both isomers. It clearly shows the efficiency of the identification and the sampling procedure for obtaining hydrogen bonds formed between methanol and water. Indeed, it can be seen how the configuration space is filled and the water molecules hydrogen bonded to the methanol. The calculated hydrogen bond distance $\mathrm{O}-\mathrm{O}$ in the complex is $2.844 \stackrel{\AA}{A}$, for the $\mathrm{CH}_{3} \mathrm{HO} \cdots \mathrm{H}_{2} \mathrm{O}$ isomer and $2.912 \AA$ for the $\mathrm{CH}_{3} \mathrm{OH} \cdots \mathrm{OH}_{2}$ isomer. For comparison, in the (1:1) liquid case, the average 

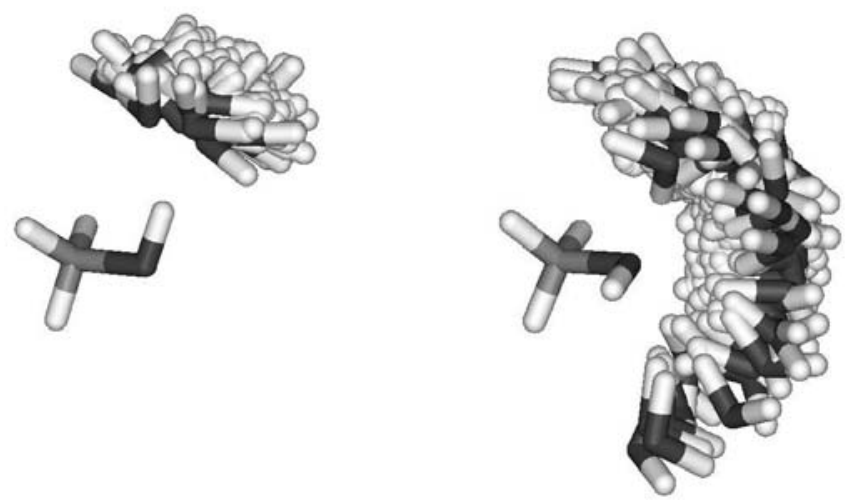

Figure 4: Superposition of configurations of the hydrogen bonds formed by methanol in aqueous solution.

distances are calculated as $2.84 \pm 0.16 \AA$ and $2.84 \pm 0.15 \AA$, respectively.

\subsection{Quantum Mechanical Calculations}

The major interest of this paper is the calculation of the binding energy of methanol in liquid water and a comparison with the result obtained for the optimized (1:1) cluster. All binding energies are obtained using counterpoise correction[32] to basis set superposition error (BSSE), i.e.;

$$
\Delta E_{A B}^{C P}=E_{A B}-E_{(A) B}-E_{A(B)}
$$

where, as usual, $E_{A B}$ is the energy of the complex, $E_{A(B)}$ is the energy of the monomer $A$ obtained with the entire basis set, including the basis set of monomer $B$, and $E_{B(A)}$ is the equivalent for the monomer $B$.

As the appropriate Boltzmann weights are included in the Metropolis Monte Carlo sampling technique the average value of the binding energy, or any other property calculated from the MC data, is given as a simple average over a chain of size $L$ of energy values:

$$
\langle E\rangle=\frac{1}{L} \sum_{i}^{L} E_{i}
$$

The quantum mechanical energies and properties are calculated individually for each of the (1:1) structures extracted from the MC simulation of the liquid and for the optimized (1:1) complexes using many-body perturbation theory in second-order with the Moller-Plesset partitioning[33], using the MP2/aug-cc-pVDZ theoretical model, implemented in the Gaussian 98 program $[34]$. 
Table 2: Calculated binding energies (kcal/mol) of the two hydrogen-bonded methanol-water isomers in gas phase. All present calculations are made using the geometry optimized with MP2/aug-cc-pVDZ. B3LYP stands for the DFT calculations of González et al[13] using the B3LYP/6-311+G(d,p) optimized geometry. ZPE stands for zero point energy difference between the complex and the separate parts using MP2/aug-cc-pVDZ.

\begin{tabular}{cccc}
\hline \hline & & & Relative \\
Method & $\mathrm{CH}_{3} \mathrm{HO} \cdots \mathrm{H}_{2} \mathrm{O}$ & $\mathrm{CH}_{3} \mathrm{OH} \cdots \mathrm{OH}_{2}$ & stability \\
\hline MP2/aug-cc-pVDZ & 5.2 & 4.5 & 0.7 \\
MP3/aug-cc-pVDZ & 4.9 & 4.4 & 0.5 \\
D-MP4/aug-cc-pVDZ & 4.8 & 4.3 & 0.5 \\
DQ-MP4/aug-cc-pVDZ & 4.7 & 4.2 & 0.5 \\
SDQ-MP4/aug-cc-pVDZ & 4.8 & 4.2 & 0.6 \\
MP4/aug-cc-pVDZ & 5.0 & 4.4 & 0.6 \\
CCSD/aug-cc-pVDZ & 4.7 & 4.2 & 0.5 \\
CCSD(T)/aug-cc-pVDZ & 5.0 & 4.5 & 0.5 \\
B3LYP/6-311+G(3df,2p) & 5.9 & 5.2 & 0.7 \\
ZPE & 1.9 & 1.6 & - \\
\hline \hline
\end{tabular}

\section{Results}

\subsection{Gas Phase Interaction}

We first consider the isolated methanol-water system, corresponding to the gas phase physical circumstance. A full geometry optimization is performed for both isomers using the second-order MP2 and the aug-cc-pVDZ basis set. Figure 1 shows the optimized hydrogen bond $\mathrm{O}-\mathrm{O}$ distance in both cases. We can note that the $\mathrm{CH}_{3} \mathrm{HO} \cdots \mathrm{H}_{2} \mathrm{O}$ isomer has a shorter distance indicating a possible stronger bond. The calculated $\mathrm{O}-\mathrm{O}$ hydrogen bond distances of $2.844 \AA$ and $2.912 \AA$ are in good agreement with the previous result[13] of $2.851 \AA$ and $2.901 \AA$. Table 2 shows the calculated results for the binding energy of both isomers after taking into account the basis-set superposition error via counterpoise correction. To analyze the role of electron correlation effects in more detail higher-order calculations, up to the coupled-cluster $\operatorname{CCSD}(\mathrm{T})$ level, were performed in the geometries obtained at the MP2/augcc-pVDZ level. At the MP2 level the $\mathrm{CH}_{3} \mathrm{HO} \cdots \mathrm{H}_{2} \mathrm{O}$ isomer is more stable by $0.7 \mathrm{kcal} / \mathrm{mol}$. This is in good agreement with previous density-functional 
theory calculations by González et al[13] that obtained the same value for the relative stability of this isomer. With all theoretical results shown in table 2 we can see that the $\mathrm{CH}_{3} \mathrm{HO} \cdots \mathrm{H}_{2} \mathrm{O}$ isomer is more stable but the relative stability is less than $1 \mathrm{kcal} / \mathrm{mol}$. In fact at the highest level used here, CCSD(T)/aug-cc-pVDZ//MP2/aug-cc-pVDZ we obtain a relative stability of $0.5 \mathrm{kcal} / \mathrm{mol}$. This agreement between MP2 and CCSD $(\mathrm{T})$ corroborates that MP2/aug-cc-pVDZ is a good theoretical model for obtaining hydrogen bond interaction. If we take into account the zero-point energy correction that is important in the gas phase results, the relative stability decreases to $0.5 \mathrm{kcal} / \mathrm{mol}(\mathrm{MP} 2)$ and $0.2 \mathrm{kcal} / \mathrm{mol}(\mathrm{CCSD}(\mathrm{T}))$. It is clear from these results that high-level ab initio calculations systematically predict the existence of both isomers with the $\mathrm{CH}_{3} \mathrm{HO} \cdots \mathrm{H}_{2} \mathrm{O}$ being the most stable. The relative stability is, however, very small being calculated to be less than $1 \mathrm{kcal} / \mathrm{mol}$ and with the best result indicating a relative stability of the $\mathrm{CH}_{3} \mathrm{HO} \cdot \mathrm{H}_{2} \mathrm{O}$ isomer by only $0.5 \mathrm{kcal} / \mathrm{mol}$.

\subsection{Liquid (1:1) Interaction}

Now we discuss the results obtained using the structures generated by the $\mathrm{MC}$ simulation at room temperature. The calculated average binding energies obtained using the (1:1) structures of the MC simulation, sampled according to the statistical correlation, as discussed above, are shown in table 3. These average results include counterpoise correction to basis set superposition error. The results show that the binding energies are the same within $0.01 \mathrm{kcal} / \mathrm{mol}$ that is much smaller than the standard deviation of the calculations. The $\mathrm{CH}_{3} \mathrm{HO} \cdots \mathrm{H}_{2} \mathrm{O}$ isomer has an average binding energy of $3.05 \pm 1.09 \mathrm{kcal} / \mathrm{mol}$ compared to the value of $3.04 \pm 0.64 \mathrm{kcal} / \mathrm{mol}$ for the $\mathrm{CH}_{3} \mathrm{OH} \cdots \mathrm{OH}_{2}$ isomer. It is clear that in the liquid situation at room temperature these results are similar and favor the conclusion that the two possible isomers are equally probable energetically. The largest standard deviation $(1.09 \mathrm{kcal} / \mathrm{mol}$ vs. $0.64 \mathrm{kcal} / \mathrm{mol})$ obtained for the $\mathrm{CH}_{3} \mathrm{OH} \cdots \mathrm{H}_{2} \mathrm{O}$ isomer is a consequence of the larger structural disorder as can be seen in figure 4. The two distributions of calculated values are shown in figure 5 . Another aspect worth mentioning is the decrease in binding energy for the liquid situation. As a result of the thermal fluctuation and the ensemble of possible configurations in the liquid we can note that the final binding energy is reduced to $60-70 \%$ of the corresponding value for the minimum-energy configuration of the gas phase. Table 3 also gives the calculated dipole moments. 
Table 3: Calculated binding energy $(\mathrm{kcal} / \mathrm{mol}$ ) of the two isomers of hydrogen-bonded methanol-water obtained with MP2/aug-cc-pVDZ including counterpoise correction to BSSE. Results shown for the liquid are averages. Dipole moments are in Debye and dipole polarizabilities (average and anisotropic) are in a.u. Rayleigh depolarizations ( $\sigma$ for normal, planar and circular polarizations) are dimensionless. Standard deviations are also shown.

\begin{tabular}{ccccc}
\hline \hline & \multicolumn{2}{c}{$\mathrm{CH}_{3} \mathrm{HO} \cdots \mathrm{H}_{2} \mathrm{O}$} & \multicolumn{2}{c}{$\mathrm{CH}_{3} \mathrm{OH} \cdots \mathrm{OH}_{2}$} \\
& liquid & gas & liquid & gas \\
\hline$\Delta \mathrm{E}$ & $3.05 \pm 1.09$ & 5.15 & $3.04 \pm 0.64$ & 4.48 \\
$\mu$ & $3.68 \pm 0.34$ & 2.04 & $3.42 \pm 0.29$ & 2.73 \\
$\alpha$ & $31.45 \pm 0.09$ & 31.19 & $31.27 \pm 0.18$ & 30.67 \\
$\Delta \alpha$ & $5.72 \pm 0.74$ & 5.90 & $5.69 \pm 1.1$ & 5.25 \\
$\sigma_{N}\left(10^{-3}\right)$ & $4.5 \pm 1.1$ & 4.7 & $4.5 \pm 1.8$ & 4.0 \\
$\sigma_{P}\left(10^{-3}\right)$ & $2.2 \pm 0.6$ & 2.4 & $2.3 \pm 0.9$ & 2.0 \\
$\sigma_{C}\left(10^{-3}\right)$ & $4.5 \pm 1.1$ & 4.7 & $4.6 \pm 1.8$ & 4.0 \\
\hline \hline
\end{tabular}

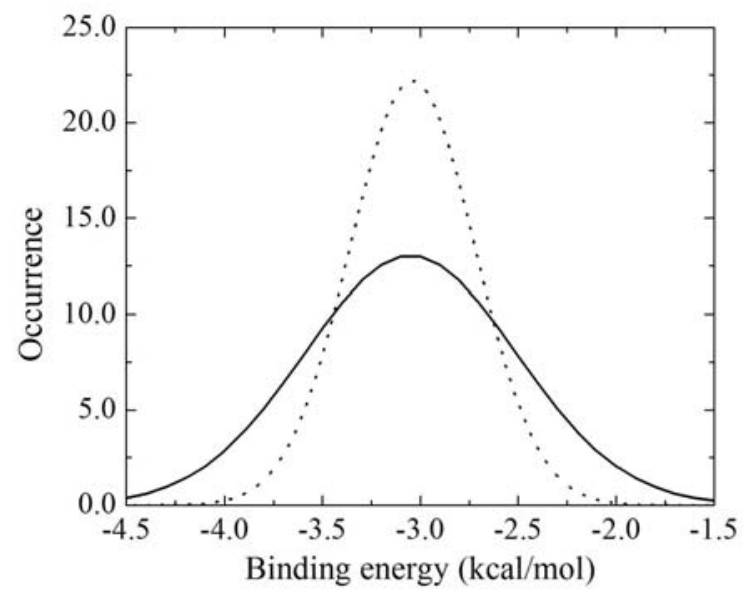

Figure 5: Histogram showing the distribution of binding energies between methanol and water. Full line is for $\mathrm{CH}_{3} \mathrm{HO} \cdots \mathrm{H}_{2} \mathrm{O}$ and dashed line is for $\mathrm{CH}_{3} \mathrm{OH} \cdots \mathrm{OH}_{2}$. 
As it can be seen the average dipole moments for the (1:1) liquid situation is considerably larger than in the (1:1) optimized complex. For completeness, table 3 also gives the average and anisotropic dipole polarizabilities of the (1:1) structures of the liquid as compared to the gas phase situation. The inhomogeneity in the electronic distribution leads to depolarization of the light elastically scattered and this Rayleigh depolarization is obtained from the calculated (1:1) dipole polarizabilities. Upon hydrogen bond formation in the aqueous environment the depolarization ratios become a distribution with the average value being only slightly decreased with respect to the value obtained for the cluster-optimized. The depolarization ratios are essentially the same for both isomers. The table 3 gives the corresponding Rayleigh depolarization ratios for different light polarizations (normal, planar and circular). Rayleigh depolarizations have been analyzed before for the case of pyridine in water with similar results as those found here[20].

\subsection{Statistical Convergence}

The efficient sampling of configurations from the simulation is crucial to ensure fast statistical convergence of the subsequent quantum mechanical calculations. Instead of performing a quantum mechanical calculation on every configuration generated by the MC simulation, as discussed in section 2.1, we used the interval of statistical correlation $[27,35]$, to select the configurations that are statistically relevant[25]. We have shown previously that performing average over thousands of successive configurations generated in the MC simulation, gives the same result as averaging over only a few statistically uncorrelated configurations[36, 37]. Using the auto-correlation function of the energy is a very efficient way to sample configurations and ensure statistically converged results. Indeed, figure 6 shows the calculated average binding energy for increased number of (1:1) configurations used in the liquid case. The results are clearly converged demonstrating the efficiency of our sampling procedure and the reliability of our average results.

\section{Final Remarks and Conclusions}

Hydrogen bond is a fundamental interaction to understand the thermodynamics of protic liquids, liquid mixtures and several biological phenomena that can only occur in the liquid environment. Hydrogen bonds between 


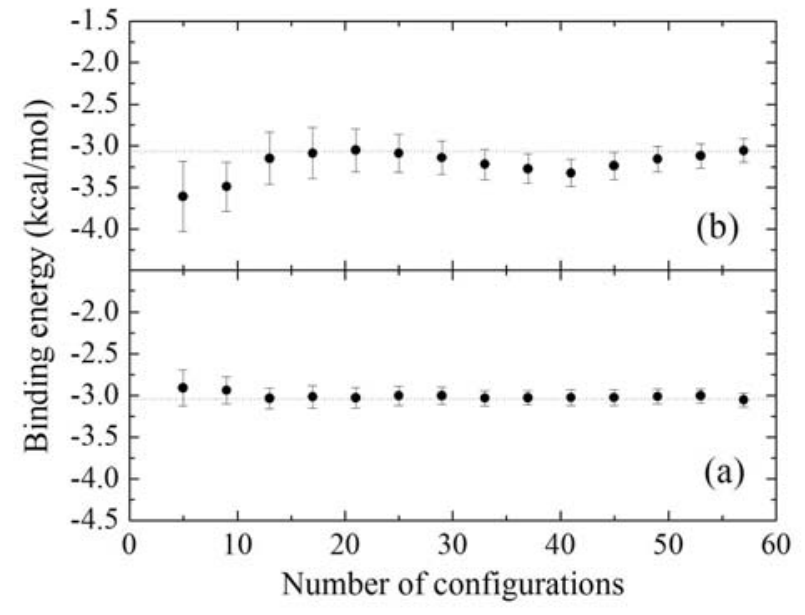

Figure 6: Convergence of the calculated binding energies for hydrogenbonded methanol-water. $(a)$ is $\mathrm{CH}_{3} \mathrm{OH} \cdots \mathrm{OH}_{2}$ and $(b)$ is $\mathrm{CH}_{3} \mathrm{HO} \cdots \mathrm{H}_{2} \mathrm{O}$. Statistical errors are shown.

bio-molecules are perhaps the most significant process regulating functions in living systems. Most understanding of the electronic structure of hydrogen bonds has been derived from studies in optimized complexes. However, the situation found in such clusters is certainly not the same as that in a liquid. Whereas in a cluster the hydrogen bond structure is fixed by a minimum-energy condition, in the liquid environment there are several configurations given by the temperature and the related natural disorder of the liquid. Therefore the hydrogen-bonded site of a solute molecule in aqueous environment experiences a great local thermal disorder. Understanding the nature of hydrogen bonds formed between a solute organic molecule and water is crucial for describing several solvation and bio-molecular processes[38]. One system that is very important is the alcohol-water mixture. Methanolwater is among the simplest of these mixtures and we have focused on the adequate treatment of the two possible isomers. In this study we considered the situation of methanol in water in conditions of extreme dilution: one methanol molecule is surrounded by bulk water. This simplified condition precludes the existence of more diversified cluster formation and makes it easier to compare with the (1:1) gas phase result.Using liquid structures generated by realistic Monte Carlo simulations, we calculated the average 
interaction energy between methanol and water using first principle calculations at the second-order perturbation theory level, MP2/aug-cc-pVDZ, and counterpoise correction to basis set superposition. We find that the statistically converged binding energies of (1:1) complexes decrease to about $2 / 3$ compared to the minimum-energy structures and that in the liquid environment the two isomers have the same average binding energy.

\section{Acknowledgments}

This paper is dedicated to Prof. Osvaldo Goscinski on the occasion of his 65th anniversary. SC, in particular, takes great pride in having been one of his graduate student and to have the opportunity to pay this modest tribute to a dear friend. This work has been partially supported by CNPq, CAPES and FAPESP (Brazil).

\section{References}

[1] F. Franks and J. E. Desnoyers, in Water Science Reviews, vol. 1, F. Franks (ed.) p. 171, Cambridge University Press (1985).

[2] F. Franks and D. J. G. Ives, Q. Rev. 20, 1 (1966).

[3] A. K. Soper and J. L. Finney, Phys. Rev. Lett. 71, 4346 (1993).

[4] S. Dixit, J. Crain, W. C. K. Poon, J. L. Finney and A. K. Soper, Nature 416, 829 (2002).

[5] J. -H. Guo et al. Phys. Rev. Lett. 91, 157401 (2003).

[6] J. E. Del Bene, J. Chem. Phys. 55, 4633 (1971).

[7] Y. C. Tse, M. D. Newton and L. C. Allen, Chem. Phys. Lett. 75, 350 (1980).

[8] G. Bolis, E. Clementi, D. H. Wertz, H. A. Scheraga and C. Tosi, J. Am. Chem. Soc. 105, 355 (1983).

[9] S. Kim, M. S. Jho and H. A. Scheraga, J. Phys. Chem. 92, 7216 (1988). 
[10] N. Bakkas, Y. Bouteiller, A. Loutellier, J. P. Perchard and S. Racine, J. Chem. Phys. 99, 3335 (1993).

[11] F. Huisken and M. Stemmler, Chem. Phys. Lett. 180, 332 (1991).

[12] P. A. Stockman, G. A. Blakee, F. J. Lovas and R. D. Suenram, J. Chem. Phys. 107, 3782 (1997).

[13] L. González, O. Mó and M. Yáñez, J. Chem. Phys. 109, 139 (1998).

[14] E. E. Fileti, R. Rivelino and S. Canuto, J. Phys. B. At. Mol. Opt. Phys. 36, 399 (2003).

[15] J. L. Finney, D. T. Bowron and A. K. Soper, J. Phys. Condens. Matter. 12, A123 (2000).

[16] G. Palinkas, E. Hawlicka and K. Heinzeger, Chem. Phys. 158, 65 (1991).

[17] H. Tanaka and K. Gubbins, J. Chem. Phys. 97, 2626 (1992).

[18] A. Laaksonen, P. G. Kusalik and I. M. Svishchev, J. Phys. Chem. A 101, 5910 (1997).

[19] T. Malaspina, K. Coutinho and S. Canuto, J. Chem. Phys. 117, 1692 (2002).

[20] E. E. Fileti, K. Coutinho, T. Malaspina and S. Canuto, Phys. Rev. E 67, 61504 (2003).

[21] M. P. Allen and D. J. Tildesley, Computer Simulation of Liquids, Oxford University Press, Oxford (1987).

[22] K. Coutinho and S. Canuto, DICE (version 2.8): A general Monte Carlo program for liquid simulation, University of São Paulo (2000).

[23] H. J. C. Berendsen, J. P. M. Postma W. F. van Gunsteren, in: B. Pullman (Ed.), Intermolecular Forces, Reidel, Dordrecht, 1981, p. 331.

[24] W. L. Jorgensen and D. S. Maxwell and J. T. Rives, J. Am. Chem. Soc. 118, 11225 (1996).

[25] K. Coutinho and S. Canuto, J. Mol. Struct. Theochem 632, 235 (2003). 
[26] S. Canuto and K. Coutinho, Int. J. Quantum Chem. 77, 192 (2000).

[27] K. Coutinho, S. Canuto and M. C. Zerner, J. Chem. Phys. 112, 9874 (2000).

[28] H. Sato and F. Hirata, J. Chem. Phys. 111, 8545 (1999).

[29] F. H. Stilinger, Adv. Chem. Phys. 31, 1 (1975).

[30] M. Mezei and D. L. Beveridge, J. Chem. Phys. 74, 622 (1981).

[31] W. L. Jorgensen, J. M. Briggs and M. L. Contreras, J. Phys. Chem. 94, 1683 (1990).

[32] S. F. Boys and F. Bernardi, Mol. Phys. 19, 553 (1970).

[33] R. J. Bartlett J. Phys. Chem. 93, 1697 (1989).

[34] M. J. Frisch, et al. Gaussian 98, Revision A.6, Gaussian, Inc., Pittsburgh PA, 1998.

[35] K. Coutinho and S. Canuto, Adv. Quantum. Chem. 28, 89 (1997).

[36] K. Coutinho, M. J. de Oliveira and S. Canuto, Int. J. Quantum Chem. 66, 249 (1998).

[37] W. R. Rocha, K. Coutinho, W. B. de Almeida and S. Canuto, Chem. Phys. Lett. 335, 127 (2001).

[38] T. W. Bell, A. B. Kkasanov and M. G. B. Drew, J. Am. Chem. Soc. 335, 127 (2001). 